\title{
Atypical Targetoid Lesions as the Presenting Sign of Myeloid Sarcoma
}

\author{
Eduardo Rodriguez, MD¹, Edward Visnaw, BS ${ }^{1}$, Frank Petr, MD², Huma Siddiqui, MD² \\ 1 University of Texas Health Science Center at San Antonio, San Antonio, TX \\ 2 South Texas Veterans Health Care System, San Antonio, TX
}

\section{ABSTRACT}

Myeloid sarcoma (MS), also known as chloroma or granulocytic sarcoma, is a rare extramedullary tumor mass that commonly occurs in acute myeloid leukemia (AML). MS may occur before other clinical signs of AML, or alongside other known AML symptoms. MS can also appear in isolation, which makes diagnosis challenging. Clinical knowledge about a typical presentation of myeloid sarcoma is limited, given the heterogeneity in the location and size of MS lesions. We describe a patient with a history of AML who developed an eruption of atypical targetoid purpuric lesions. Diagnostic workup, including laboratory and histologic specimen from the skin were consistent with MS. MS should be suspected in a patient with a history of myeloproliferative disorder who develops atypical targetoid purpuric papules.

\section{INTRODUCTION}

Myeloid sarcoma (MS), also known as chloroma or granulocytic sarcoma, is a rare extramedullary solid tumor composed of immature myeloid cells. MS most commonly consists of myeloblasts, with or without features of promyelocytic or neutrophilic maturation, which partially or totally efface the tissue architecture ${ }^{1-2}$. MS commonly occurs in association with acute myeloid leukemia (AML), affecting 2 to $7 \%$ of these patients $^{3-4}$. MS can also manifest in patients with a history of chromosomal abnormalities, myeloproliferative disorders, myelodysplastic syndrome, or in a blast phase of chronic myeloid leukemia. It may occur before other clinical signs of AML, or alongside other known AML symptoms. MS can also appear in the absence of a primary systemic disease and predate the onset of an underlying hematologic malignancy². Additionally, MS can present as the initial manifestation of relapse in an already treated case or as a therapy related neoplasm ${ }^{1-3}$. MS has a slight male predominance and can occur at any age. Although it can present at any site of the body, the most common reported sites are the skin, lymph nodes, testes, bone, peritoneum, and gastrointestinal tract ${ }^{2-5}$. CD68-KP1 is the most commonly expressed marker in MS followed by myeloperoxidase (MPO), CD117, CD99, CD68/ PG-M1, lysozyme, CD34, terminal deoxynucleotidyl transferase (TdT), CD56, CD61/linker of activated $\mathrm{T}$ lymphocyte/factor VIII-related antigen, CD30, glycophorin A, and CD4. ${ }^{2}$ In this report, we present an unusual appearance of MS presenting with atypical targetoid lesions in a patient undergoing treatment for AML.

November $2021 \quad$ Volume 5 Issue 6 


\section{CASE REPORT}

A 73-year-old male, with a history of AML on azacitidine and enasidenib, presented to the dermatology clinic for evaluation of a purpuric eruption to the arms and legs of 7 to 10 days duration. Review of systems were otherwise negative, and the patient had no further complaints at the time. Physical exam revealed scattered, round, purpuric macular lesions with a firm, smooth, central, purpuric papule present on the left medial upper arm, right medial thigh, left upper thigh, popliteal fossa, and lower legs/ankle region (Figure 1).

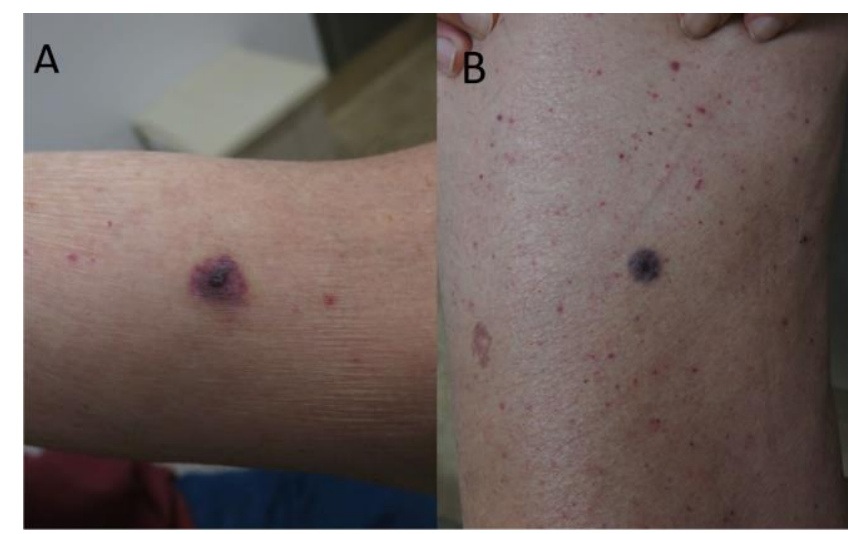

Figure 1. A) Round violaceous macules with central papule to left upper arm and B) right anterior thigh respectively.

At the time of patient presentation, laboratory evaluation was significant for anemia (Hemoglobin $8.4 \mathrm{~g} / \mathrm{dL}$ [Reference range: $13.5-17.5 \mathrm{~g} / \mathrm{dL}]$ ), thrombocytopenia (Platelets $2310^{3} / \mu \mathrm{L}$ [Reference range: 150$\left.40010^{3} / \mu \mathrm{L}\right]$ ) elevated absolute lymphocytes (Lymphocytes, alternate absolute $6.010^{3} / \mu \mathrm{L}$

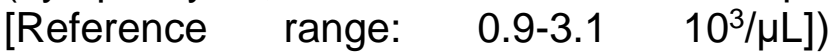
monocytes (Monocytes $1.6 \quad 10^{3} / \mu \mathrm{L}$ [Reference range: $0.2-0.810^{3} / \mu \mathrm{L}$ ]), and blast cell counts (Blasts 6.1\% [Reference range: $0 \%])$. The patient also presented with minimally elevated lactate dehydrogenase (Lactate dehydrogenase 279 IU/L
[Reference range 140-271 IU/L]) and serum uric acid (Serum uric acid $5.0 \mathrm{mg} / \mathrm{dL}$ [2.3$7.6 \mathrm{mg} / \mathrm{dL}])$.

A punch biopsy of the right thigh lesion was performed, demonstrating superficial, deep perivascular, and periadnexal mononuclear infiltrate with occasional atypical mitoses. By immunohistochemistry, this infiltrate expressed CD117, CD43, CD4, focal CD34, focal myeloperoxidase, and was negative for CD3 and CD20.(Figures $2 \& 3$ ) Scattered CD68+ cells were present. Special stains for organisms were negative. Bacterial and fungal tissue cultures were negative. The diagnosis of myeloid sarcoma (MS) was then made.

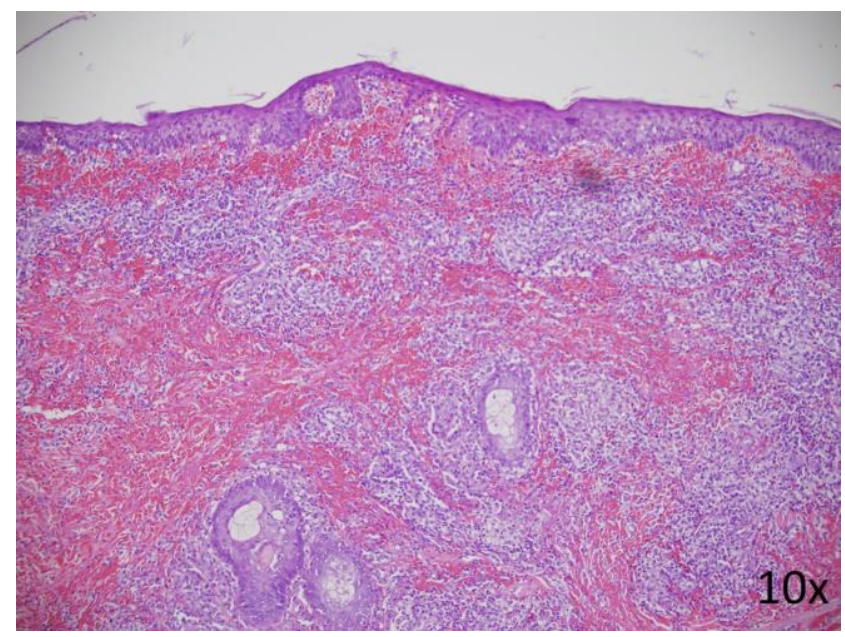

Figure 2. Punch Biopsy, Right Thigh, H\&E; Superficial and deep perivascular and periadnexal mononuclear infiltrate with occasional atypical mitoses.

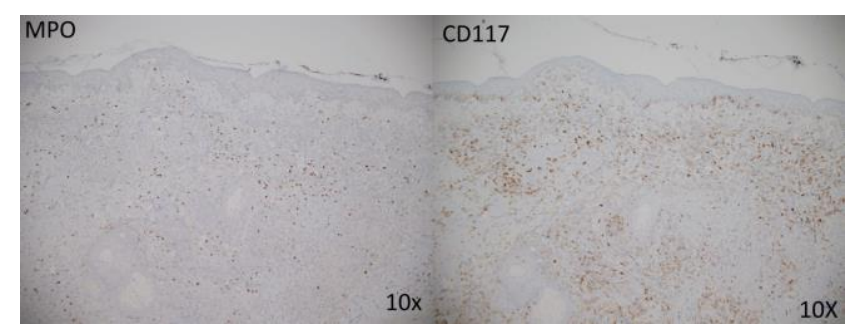

Figure 3. Punch Biopsy, Right Thigh, IHC MPO \& CD117. Scattered MPO positive staining cells along with numerous CD117 positive staining cells throughout the dermis.

November $2021 \quad$ Volume 5 Issue 6 


\section{DISCUSSION}

Clinical knowledge about a typical presentation of myeloid sarcoma is limited, given the heterogeneity in the location and size of MS lesions. Patients with MS are typically younger, have a higher white cell count, and have a greater proportion of AML with monocytic differentiation ${ }^{6}$. The presenting symptom of MS is typically due to the mass effect of the tumor on nearby tissues or organ dysfunction due to infiltration ${ }^{2,4}$. Therefore any new cutaneous lesion in a patient with AML should raise suspicion for MS. Cutaneous MS typically presents as multiple papules, plaques, or nodules ${ }^{4}$. The most common region involved is the torso, although head, neck, and extremities are also commonly involved? ${ }^{7}$. Diagnosing MS depends on a combination of clinical features, radiological evidence, and in most cases tissue biopsy ${ }^{1}$. Most common imaging modalities include CT and MRI to exclude differential diagnoses such as abscesses and hematomas. MRI is preferred in the setting of musculoskeletal lesions and involvement of the central nervous system. PET-CT has also been reported to be helpful in localization of tumors and detection of synchronous lesions. ${ }^{6}$ MS has been reported to demonstrate sensitivity to different therapies such as chemotherapy, radiation, and hematopoietic stem cell transplantation. Optimal treatments for MS have not been established due to lack of randomized controlled trials, however, chemotherapy is commonly the initial therapy. Other treatment methods that have been reported include local therapies such as surgery, radiotherapy, or a combination of both. However, retrospective series have demonstrated a high rate of progression to AML in patients with isolated MS treated with local therapies only. No controlled trials evaluating the role of allogenic hematopoietic stem cell transplantation in patients with isolated MS have been performed but retrospective studies have demonstrated encouraging results. ${ }^{6}$ MS prognosis can be variable depending on the different backgrounds of MS formation but it is typically accepted to be poor. Untreated MS commonly transforms to AML over a 1012-month period. ${ }^{6,}$ 8-10 Patients with isolated MS involvement of the pelvis, eyes, gonads, genitourinary organs, and gastrointestinal mucosa had reported better outcomes as compared to those with involvement of primary soft tissue sites, nervous system, lymphatic, and hematopoietic tissues. In prior studies, patients with MS had a greater 3-year survival rate as compared to patients with non-MS AML. ${ }^{11}$

\section{CONCLUSION}

This case represents an atypical presentation of myeloid sarcoma in a patient with known acute myeloid leukemia. Despite multiple reports in the literature, the authors have found no other reported cases of MS presenting with atypical targetoid lesions. ${ }^{12-}$ ${ }^{13}$ The atypical targetoid presentation in this patient emphasizes the importance of having a high index of suspicion for MS when a patient with a history of a myeloproliferative disorder presents with any new suspicious cutaneous lesions or eruptions. A thorough work-up should be performed when patients present with new widespread cutaneous lesions, especially on patients who have current or prior history of any hematologic malignancies. A representative lesion biopsy with immunohistochemistry evaluation for assessment of features consistent with MS should be highly considered on these patients. 


\section{Conflict of Interest Disclosures: None}

Funding: None

\section{Corresponding Author:}

Eduardo Rodriguez, MD

7979 Wurzbach Road

San Antonio, Texas 78229

Phone: $210-450-9840$

Email: rodrigueze25@uthscsa.edu

\section{References:}

1. Meyer H, Beimler M, Borte G, Pönisch W, Surov A. Radiological and Clinical Patterns of Myeloid Sarcoma. Radiology and Oncology. 2019;53(2):213-218. doi: 10.2478/raon-20190014.

2. Campidelli C, Agostinelli C, Stitson R, Pileri SA. Myeloid sarcoma: extramedullary manifestation of myeloid disorders. American journal of clinical pathology. 2009;132(3):426-

3. Kaur V, Swami A, Alapat D, et al. Clinical characteristics, molecular profile and outcomes of myeloid sarcoma: a single institution experience over 13 years. Hematology. 2018;23(1):17-24. doi:10.1080/10245332.2017.1333275

4. Neiman RS, Barcos M, Berard C, et al. Granulocytic sarcoma: A clinicopathologic study of 61 biopsied cases. Cancer. 48(6):1426-1437. doi:10.1002/10970142(19810915)48:6<1426::AIDCNCR2820480626>3.0.CO;2-G

5. Kawamoto K, Takizawa J, Sone H, Miyoshi $\mathrm{H}$, Yoshida N, Ohshima K. Clinicopathological, cytogenetic, and prognostic analysis of 131 myeloid sarcoma patients. American Journal of Surgical Pathology. 40(11):1473-1483. doi:10.1097/PAS.0000000000000727

6. Almond LM, Charalampakis M, Ford SJ, Gourevitch D, Desai A. Myeloid Sarcoma: Presentation, Diagnosis, and Treatment. Clinical Lymphoma, Myeloma \& Leukemia. 2017;17(5):263-267. doi: 10.1016/j.clml.2017.02.027.

7. Klco JM, Welch JS, Nguyen TT, et al. State of the art in myeloid sarcoma. International Journal of Laboratory Hematology. 2011;33(6):555-565. doi: 10.1111/j.1751553X.2011.01361.x
8. Katagiri T, Ushiki T, Masuko M, et al. Successful 5-azacytidine treatment of myeloid sarcoma and leukemia cutis associated with myelodysplastic syndrome: A case report and literature review. Medicine. 2017;96(36):e7975. doi:10.1097/MD.0000000000007975

9. Antic D, Elezovic I, Milic N, et al. Is there a "gold" standard treatment for patients with isolated myeloid sarcoma? Biomedicine \& Pharmacotherapy. 2013;67(1):72-77. doi:10.1016/j.biopha.2012.10.014

10. Kawamoto K, Miyoshi H, Yoshida N, Takizawa J, Sone H, Ohshima K. Clinicopathological, Cytogenetic, and Prognostic Analysis of 131 Myeloid Sarcoma Patients. The American journal of surgical pathology. 2016;40(11):1473-1483.

Accessed August 19, 2020.

http://search.ebscohost.com.libproxy.uthscsa .edu/login.aspx?direct=true $\& \mathrm{db}=\mathrm{cmedm} \& \mathrm{AN}$ $=27631510 \&$ site $=$ eds-live \&scope $=$ site

11. Movassaghian, M., Brunner, A. M., Blonquist, T. M., Sadrzadeh, H., Bhatia, A., Perry, A. M., Attar, E. C., Amrein, P. C., Ballen, K. K., Neuberg, D. S., \& Fathi, A. T. (2014).

Presentation and outcomes among patients with isolated myeloid sarcoma: a Surveillance, Epidemiology, and End Results database analysis. Leukemia \& Lymphoma, 56(6), 1698-1703.

https://doi.org/10.3109/10428194.2014.9630 80

12. Niiyama $S$, Amoh $Y$, Watarai A, Katsuoka K, Mukai $\mathrm{H}$. Cutaneous myeloid sarcoma presenting as grey pigmented macules. Acta dermato-venereologica. 2012;92(6):629-630. doi:10.2340/00015555-1312

13. Hurley MY, Ghahramani GK, Frisch S, et al. Cutaneous Myeloid Sarcoma: Natural History and Biology of an Uncommon Manifestation of Acute Myeloid Leukemia. Acta DermatoVenereologica. 2013;93(3):319-324. doi:10.2340/00015555-1458 